Secondly, other, as yet unidentified, mutations in the mt genome of the patients could have a modulatory effect on the phenotypic expression of the thymine deletion. PCRSSCP analysis of all other tRNA genes did not show additional mtDNA variations, but the complete sequence of the mt genome was not determined. Therefore, the presence of an additional heteroplasmic mtDNA mutation cannot be excluded.

Thirdly, the mt genome is known to be highly polymorphic. Therefore, the deletion could be classified as a very uncommon genetic polymorphism, and its presence in the two families suffering from $\mathrm{mt}$ disorders is no more than a striking coincidence.

We screened, along with the two families, more than 70 people (both patients and controls) for mutations in the same DNA fragment. Five different nucleotide substitutions, at positions $15904 \mathrm{C}$ to $\mathrm{T}, 15907 \mathrm{~A}$ to $G, 15924 A$ to $G, 15928 \mathrm{G}$ to $A$ in tRNA ${ }^{\text {thr }}$ (all previously reported), and $16017 \mathrm{~T}$ to $\mathrm{C}$ in tRNA ${ }^{\text {pro }}$, were identified. ${ }^{56}$ All sequence variations were homoplasmic and observed both in patients and control mtDNA. The tRNA ${ }^{\text {thr }}$ gene also harbours several nucleotide alterations responsible for $\mathrm{mt}$ encephalomyopathies: a $\mathrm{G}$ to A transition at nucleotide 15915 , an $A$ to $G$ point mutation at nucleotide 15923, and a $G$ to A mutation at bp $15927 . .^{5}$ This particular DNA fragment seems to represent a "hot spot" for DNA variations in the mt genome.

This report illustrates the difficulties in diagnosing $\mathrm{mt}$ disorders. More pedigrees with this deletion mutation will need to be characterised to understand the real significance of this DNA aberration. In addition, micro-rearrangements involving tRNA genes should be considered as possible causes for oxidative phosphorylation diseases, even if they are homoplasmic in both patients and unaffected relatives.

This work was supported by the National Fund for Scientific Research Flanders (NFWO) 3.0065.87, the Fund for Scientific Research Flanders (FWO Vlaanderen) grant G.0181.98, and the Research Council of the Vrije Universiteit Brussel (VUB) (Brussels, Belgium).

SARA SENECA WILLY LISSENS INGE LIEBAERS

Department of Medical Genetics, Academic Hospital Vrije Universiteit, B-1090 Brussels, Belgium

PETER VAN DEN BERGH

Laboratory of Neuropathology, Department of Neurology, University Hospital Saint-Luc Université Catholique de Louvain, Brussels, Belgium

MARIE CECILE NASSOGNE

Department of Paediatric Neurology, University Hospital Saint-Luc Université Catholique de Louvain Brussels, Belgium

AVRAM BENATAR

Department of Paediatric Cardiology, Academic Hospital Vrije Universiteit Brussel, Brussels, Belgium

LINDA DE MEIRLEIR

Department of Paediatric Neurology, Academic Hospital Vrije Universiteit Brussel, Brussels, Belgium

1 Sprinzl M, Steegborn C, Hübel F, Steinberg S. Compilation of tRNA sequences of tRNA genes. Nucleic Acids Res 1996;24:68-72.

2 Tiranti V, Chariot P, Carella F, et al. Maternally inherited hearing loss, ataxia and myoclonus associated with a novel point mutation in mitoassociated with a novel point mutation in mito-
chondrial tRNA ${ }^{\text {Ser(UCN) }}$ gene. Hum Mol Genet chondrial tRNA

3 Santorelli F, Tanji K, Sano M, et al. Maternally inherited encephalopathy associated with a single-base insertion in the mitochondrial tRNA $^{\text {Trp }}$ gene. Ann Neurol 1997;42:256-60.
4 Shoffner JM, Bialer MG, Pavlakis SG, et al. Mitochondrial encephalomyopathy associated with a single nucleotide pair deletion in the with a single nucleotide pair deletion in the
mitochondrial tRNA ${ }^{\mathrm{Leu}(U \mathrm{UR})}$ gene. Neurology mitochondrial

5 Thomas AW, Edwards A, Sherratt EJ, Majid A, Gagg J, Alcolado JC. Molecular scanning of candidate mitochondrial tRNA genes in type (non-insulin dependent) diabetes mellitus. $\mathcal{f}$ Med Genet 1996;33:253-5.

6 Houshmand M, Larsson NG, Holme E, Oldfors A, Tulinius $\mathrm{MH}$, Andersen $\mathrm{O}$. Automatic A, Tulinius $\mathrm{MH}$, Andersen O. Automatic patients with mitochondrial encephalomyopathy. Biochim Biophys Acta 1994;1226:49-55.

7 Nishino I, Seki A, Maegaki Y, et al. A novel mutation in the mitochondrial tRNA(Thr) gene associated with a mitochondrial encephalomyopathy. Biochem Biophys Res Comm 1996; 225:180-5.

\section{Severe primary pulmonary hypoplasia ("acinar dysplasia") in sibs: a genetically determined mesodermal defect?}

We report on two sisters who died neonatally from severe pulmonary hypoplasia withou obvious cause. It was not associated with any other pathological condition, excep mild growth retardation. Histological examination of the lungs showed greatly reduced alveolar parenchyma, with almost complete absence of mature alveoli. The amount of interstitial connective tissue was increased and the bronchial cartilage plates appeared dysplastic. This form of severe pulmonary hypoplasia is very unusual; so far only two similar cases have been reported as pulmonary "acinar dysplasia". We present the first familial occurrence of this condition and suggest autosomal recessive inheritance.

Case 1 was the first child of a young, healthy, and non-consanguineous couple. She was born at 40 weeks after an uneventful pregnancy. Clinically, there was no oligo- or polyhydramnios and the mother experienced

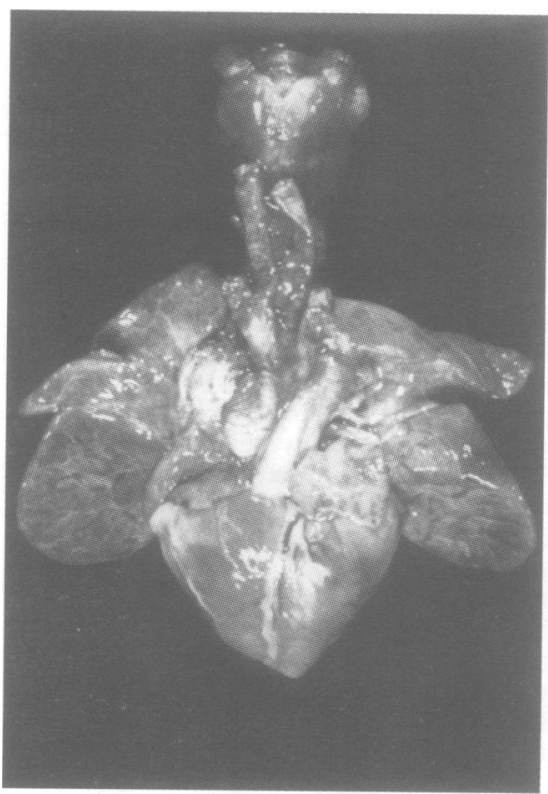

Figure 1 Thoracic organs removed as a block. Severe pulmonary hypoplasia is illustrated by the fact that the inferior surfaces of the lungs are not in line with the apex of the heart. normal fetal movements. No maternal medication or exposure to potentially teratogenic agents were recorded. Immediately aftef birth, the infant developed severe respirator distress. Chest $x$ rays and CT scans showe $₫$ marked pulmonary hypoplasia, complicate by bilateral pneumothoraces. No other radio logical abnormalities could be documente $\mathbb{\mathbb { D }}$ and the skeletal structures were normal: Despite intensive treatment, she died on the? second day.

Clinical examination showed no externaE abnormalities. Body length $(49.5 \mathrm{~cm})$ and foot length $(8.0 \mathrm{~cm})$ were normal, but bod? weight $(2860 \mathrm{~g})$ and head circumference $(32.5 \mathrm{~cm})$ were between the 10 th and 25 th centile. The karyotype was normal.

The only gross abnormality found a necropsy was pulmonary hypoplasia (fig 1) with a combined lung weight of $20.2 \mathrm{~g}$ (nor- $\omega$ $\mathrm{mal} 56 \pm 15$ ) and a lung weight/body weight ratio of 0.007 (normal $>0.012$ ). The lungso were normally lobed, but the lobular mark-w ings were accentuated owing to thickened interlobular septa. Histology of the lungs. showed extreme hypoplasia beyond the bron- 9 chiolar level with almost complete absence of ${ }^{+}$ mature alveoli and disproportionately numer-음 ous bronchi and bronchioli (fig 2). The bron-chial branching pattern was normal. The $Z$ peripheral lung parenchyma consisted merely을 of bronchioles and few immature canalicular $\frac{\mathbb{D}}{3}$ structures, separated by an increased amount of interstitial connective tissue. The loosely ㅡㅡ textured and cellular interstitial tissue con- $\vec{\theta}$ tained numerous, small, thin walled, ande dilated blood vessels and foci of extramedul-. lary haematopoiesis. The bronchial cartilage plates appeared unduly large and numerous and displayed a persistent immature cellular aspect with a pale matrix. The diaphragm and phrenic nerves were normal. The brain and $\frac{\mathrm{O}}{\mathrm{Q}}$ spinal cord were normal both grossly and microscopically. Skeletal muscle biopsies $\overline{\bar{O}}$ showed no abnormalities.

Case 2 was born at 40 weeks as the product of the mother's third pregnancy. A normalo amount of amniotic fluid was documented clinically and confirmed by ultrasound. Her

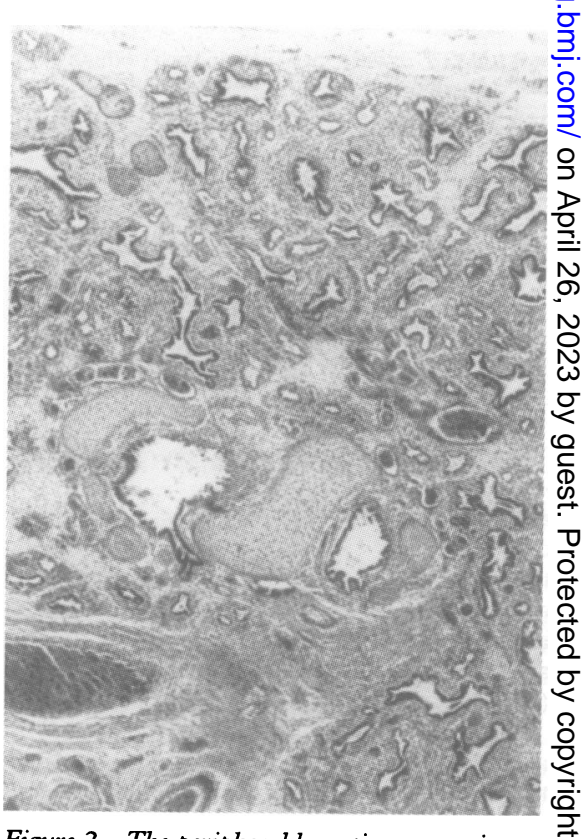

Figure 2 The peripheral lung tissue contains virtually no alveoli, but consists merely of bronchioles. The bronchial cartilage plates are unusually prominent. Note the immature and cellular ("dysplastic") aspect of the cartilaginous tissue (HE्E). 
second pregnancy ended with the birth of a healthy son. The third child died on the first day of life in severe respiratory distress. Like her sister, she was mildly growth retarded with a birth weight $(2820 \mathrm{~g})$ and head circumference $(32.5 \mathrm{~cm})$ between the 10th and 25 th centiles. Body length $(50.5 \mathrm{~cm})$ and foot length $(8.1 \mathrm{~cm})$ were normal. Clinical, radiological, and pathological findings were exactly the same as in case 1 . The combined lung weight was $18.9 \mathrm{~g}$, with a lung weight/body weight ratio of 0.0067 . The karyotype was normal.

Pulmonary hypoplasia is a common cause of intractable neonatal respiratory distress. As a rule, pulmonary hypoplasia is not a primary malformation but a secondary deformation. ${ }^{1}$ Thus, the lung anlagen and initial organogenesis are normal, until a mechanical factor interferes with development, which thereafter proceeds abnormally. Secondary pulmonary hypoplasia is usually caused by one of the following conditions: prolonged oligohydramnios, diaphragmatic anomalies, skeletal dysplasia, massive chronic pleural effusions, or neuromuscular disease. ${ }^{2}$ Primary pulmonary hypoplasia, that is, an intrinsic abnormality of the lungs with poor development from the beginning, does exist but is exceedingly rare. It is a diagnosis of exclusion that can only be made after all the known aetiologies of secondary pulmonary hypoplasia have been ruled out. This needs thorough pathological examination, including examination of the central and peripheral nervous system and skeletal muscle tissue.

To date, only a limited number of cases of primary pulmonary hypoplasia have been reported, with both sporadic ${ }^{3-5}$ and familial occurrences. ${ }^{6.9}$ There is a striking lack of uniformity in the histology of these cases. Swischuk et $a l^{4}$ and Langer and Kaufmann described reduced radial alveolar counts without other architectural abnormalities of the alveoli. Surprisingly, Frey $e t a l^{\beta}$ and $\mathrm{Hamel}^{9}$ found severely hypoplastic but histologically normal lungs with normal radial alveolar counts. In our two sibs, the lungs were normally lobed but weighed less than $50 \%$ of the expected value for the gestational age. Histological examination showed an almost complete lack of alveolar development, increased amounts of interstitial connective tissue, and abnormal (dysplastic) bronchial cartilage. While, admittedly, primary pulmonary hypoplasia is a heterogeneous condition, the two cases reported here represent a specific entity. Similar histological findings have been described in only two previous case studies. ${ }^{10}$ We agree with Ruthledge and Jensen ${ }^{10}$ and Chambers ${ }^{11}$ that this lesion represents a severe form of peripheral pulmonary maldevelopment or "acinar dysplasia", rather than pure hypoplasia. Apparently, the lung development was arrested in the early canalicular stage and development of the alveolar tissue did not take place. Stocker ${ }^{12}$ suggested that this form of pulmonary hypoplasia might be included in an expanded classification of cystic adenomatoid malformation of the lung ("type 0").

The aetiology and pathogenesis of pulmonary acinar dysplasia are unknown. The epithelium of the respiratory tract up to the alveolar lining is of foregut endodermal origin. Experimental embryological studies have shown that the sequential branching of the tracheobronchial tree is induced by the splanchnopleural mesoderm..$^{13}$ This leads to the conclusion that acinar dysplasia may be an intrinsic defect of the lung mesoderm. The observation of increased interstitial connective tissue and dysplastic bronchial cartilage in two cases appears to corroborate this. However, pulmonary development is an extremely complicated process, depending upon reciprocal epithelial-mesenchymal interactions. For instance, the developing pulmonary epithelium is the site of growth factors such as epidermal growth factor and transforming growth factor- $\alpha$, which influence differentiation of the mesenchymal compartment and overall organogenesis. More specifically, defects in lung specific transcription factors, such as thyroid specific enhancer binding protein, normally expressed in the developing pulmonary epithelium, have been shown to inhibit normal lung development, resulting in rudimentary cystic lungs. ${ }^{14}$ Thus, we cannot rule out a defect in the epithelial cell compartment as the initial cause of this malformation.

This is the first familial report of pulmonary acinar dysplasia, suggesting an autosomal recessive mode of inheritance for a gene critical for normal lung parenchymal development.

PHILIPPE MOERMAN

Department of Pathology, University Hospital St-Rafaël, Minderbroedersstraat 12, B-3000 Leuven, Belgium

CHRIS VANHOLE HUGO DEVLIEGER

Department of Paediatrics, University Hospital Gasthuisberg, Leuven, Belgium

JEAN-PIERRE FRYNS

Department of Human Genetics, University Hospital Gasthuisberg, Leuven, Belgium

1 Spranger J, Benirschke K, Hall JG, et al. Errors of morphogenesis: concepts and terms. Recommendations of an International Working Group. F Pediatr 1982;100:160-5.

2 Wigglesworth JS. Pathology of the lung in the fetus and neonate, with particular reference to problems of growth and maturation. Histopathology 1987;11:671-89.

3 Mendelsohn G, Hutchins GM. Primary pulmonary hypoplasia. Report of a case with polyhydramnios. Am $\mathcal{F}$ Dis Child 1971;131:1220-3.

4 Swischuk LE, Richardson CJ, Nichols MM, Ingman MJ. Primary pulmonary hypoplasia in the neonate. F Pediatr 1979;95:573-7.

5 Langer R, Kaufmann HJ. Primary (isolated) bilateral pulmonary hypoplasia: a comparative study of radiologic findings and autopsy results. Pediatr Radiol 1986;16:175-9.

6 Boylan P, Howe A, Gearty J, O'Brian NG. Familial pulmonary hypoplasia. Ir $\mathcal{f} \mathrm{Med} S \mathrm{Si}$ 1977;146:179-80.

7 Fraser FC. Personal communication. In McKusick VA. Mendelian inheritance in man. MIM number 265430. Baltimore: The Johns Hopkins University Press, 1992:1666.

8 Frey B, Fleishauer A, Gersbach M. Familial isolated pulmonary hypoplasia: a case report, suggesting autosomal recessive inheritance. Eur Pediatr 1994;153:460-3.

9 Hamel BCJ. Familial primary pulmonary hypoplasia. Eur $\mathcal{F}$ Pediatr 1995;154:336.

10 Ruthledge JC, Jensen P. Acinar dysplasia: a new form of pulmonary maldevelopment. Hum Pathol 1986;17:1290-3.

11 Chambers HM. Congenital acinar aplasia: an extreme form of pulmonary maldevelopment Pathology 1991;23:69-71.

12 Stocker JT. Congenital and developmental diseases. In: Dail DH, Hammar SP, eds. Pulmonary pathology. 2nd ed. New York: SpringerVerlag, 1994:155-90.

13 Wessels NK. Mammalian lung development: interactions in formation and morphogenesis of tracheal buds. F Exp Zool 1970;175:455-66.

14 Kimura S, Hara Y, Pineau T, et al. The Tlebp null mouse: thyroid-specific enhancer-binding protein is essential for the organogenesis of the thyroid, lung, ventral forebrain, and pituitary. thyroid, lung, ventral for
Genes Dev 1996;10:60-9.

\section{Coexistence of Gaucher disease type 1 and Joubert syndrome}

Joubert syndrome (JS) is an autosomal recessive disorder in which cytogenetic, biochemi$\mathrm{cal}$, and molecular markers are unknown. It is a distinct clinicopathological entity, characterised by cerebellar vermis dysgenesis, episodic tachypnoea and apnoea in the neonatal period, jerky eye movements, developmental delay, and hypotonia. Associated features may include agenesis of the corpus callosum, chorioretinal colobomata, renal cysts, congenital hepatic fibrosis, and polydactyly. ${ }^{1}$ The molecular basis of JS has recently been investigated by analysing the WNT1 gene, the human homologue of murine wnt-1, for mutations in patients with JS. It was concluded that WNT1 is not a critical gene for JS. ${ }^{2}$

In contrast to JS, the enzymatic and molecular basis in Gaucher disease (GD), an autosomal recessively transmitted lysosomal storage disorder, has been elucidated. ${ }^{3} \mathrm{GD}$ is caused by a deficiency of glucocerebrosidase activity and the gene is located in chromosome 1q21. Clinically, there are three major variants of GD classified by the age of onset, severity of the visceral symptoms, and by the presence or absence of neurological involvement: type 1, the most common variant has a non-neuronopathic course; type 2 presents in infancy with severe neurological involvement; type 3 usually presents as a severe, slowly progressive neurodegenerative disease. Recently, new neonatal and fetal subtypes with a severe course have been described. ${ }^{4}$ In this human subtype and in murine models a total disruption of the glucocerebrosidase gene caused the fulminant disease. ${ }^{5}$ More than 50 different mutations in the glucocerebrosidase gene have been identified in patients with GD, producing an enzyme which is catalytically abnormal, rapidly degraded, or truncated. Although there is a wide variation in clinical expression in GD which cannot generally be explained by the genotype alone, the four most frequently identified mutations are to some degree predictive of the course of the disease. ${ }^{36}$ For example, patients having at least one N370S mutation, whether as homozygotes or compound heterozygotes, are at a very low risk for primary neurological involvement, while those who are homozygotes for the L444P mutation usually present with neurological abnormalities. $^{36}$ In one hydrops fetus a

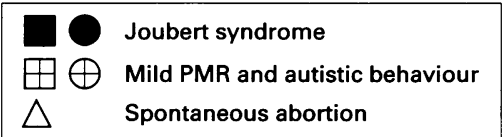

Figure 1 Pedigree of the family. Results of mutational analysis in relation to Gaucher disease type 1 are presented.

Gluc $^{*}=\beta$-glucocerebrosidase activity in fibroblasts expressed as $\mathrm{nmol} / \mathrm{h} / \mathrm{mg}$ protein (normal range 120-400); NA-not available; $P M R=$ sychomotor retardation. 